\title{
EDITORIAL
}

\section{Common ground on the critical path}

\author{
Greater collaboration between regulatory authorities, industry and academia is increasingly \\ acknowledged as the answer to some of the thorniest problems in drug development, such \\ as validation of biomarkers of efficacy and toxicity. But for such collaborative efforts to be \\ effective, two key issues need to be addressed: funding and information sharing.
}

In March 2004, the FDA released a pivotal white paper Innovation or Stagnation? Challenge and Opportunity on the Critical Path to New Medical Products ${ }^{1}$, intended to highlight the need for targeted efforts to modernize the tools, techniques and methods used to evaluate the safety and efficacy of drug products. Two years on, the Critical Path initiative has reached its next phase: 76 potential projects that the FDA believes will help accelerate the process of bringing new drugs to the market have just been announced ${ }^{2}$. Unsurprisingly, the two major themes are improving clinical trial design and approaches for the development of biomarkers for predicting toxicity and efficacy, both of which have been widely recognized by all parties as vital to improving the effectiveness of drug development.

However, despite this widespread recognition, there are two key stumbling blocks. One is funding. As highlighted in the news story this month on $\mathrm{p} 271$, how this issue could be resolved is still uncertain, as it can't just depend on the FDA alone. The FDA could potentially receive just US\$5.9 million from the President's budget this year for initiatives related to the Critical Path, and although this will be valuable in funding staff to develop guidances and for small grants to seed projects, this investment is far from the level needed for a research endeavour of such scale.

The second, potentially related, issue is the need for sharing information and expertise that industry has traditionally regarded as commercially sensitive. On this issue though, significant progress has been made since the Critical Path paper was published. Particularly notable has been the founding of the C-Path Institute (http://www.c-path.org) - a nonprofit research and education institute with the mission of facilitating Critical Path initiatives - by the FDA, the University of Arizona and SRI International. The institute is based on the model of the National Center for Food Safety and Technology (NCFST), which was set up 17 years ago by the FDA, the Illinois Institute of Technology and the food industry to foster collaboration between industry and FDA scientists on research into food safety and technology. Mirroring the NCFST, a fundamental goal of the C-Path Institute is to provide 'neutral ground' where the biopharma industry, academia and the FDA can share information and expertise needed for tackling the bottlenecks in drug development.

Indeed, companies are increasingly recognizing that some of these challenges could be more effectively addressed by the industry as a whole, rather than by each company taking its own approach. A key example is that of strategies for discovering and validating biomarkers of drug safety and efficacy (see p310 of this issue for the first in a new series of articles on biomarkers). This field has seen a flurry of investment and research activity by pharmaceutical companies in recent years, but even so, it could typically be beyond any individual company to provide the amount of data needed to validate a new biomarker. By pooling resources, the standards needed for authorities such as the FDA to accept a biomarker in regulatory decision-making could be reached faster and more costeffectively. And encouragingly, one such project at C-Path, on the development of biomarkers to predict drug safety, already has the support of several large pharmaceutical companies for an unprecedented degree of information sharing.

If successful, this pioneer project could greatly advance an area highlighted in the FDA's original white paper as one in which industry is still using decadesold, expensive and time-consuming methods, despite major technical advances, in part because there hasn't been a route for novel approaches to be approved by the regulators, or conversely, the opportunity for the regulators to suggest how old methods could be replaced. Further willingness by industry to investigate common ground with the regulatory authorities in areas such as biomarker research, coupled with a willingness to contribute somehow to the funding that is urgently required for such initiatives, could lead to the critical path to new drugs becoming much smoother and more predictable than at present.

\footnotetext{
. Innovation or Stagnation? Challenge and Opportunity on the Critical Path to New Medical Products [online], < http://www.fda.gov/oc/ initiatives/criticalpath/whitepaper.html $>$ (2004).

2. Critical Path Opportunities List [online], < http://www.fda.gov/od/ initiatives/criticalpath/reports/opp_list.pdf > (2006).
} 